\section{A nurse led clinic and computer decision support system for anticoagulation decisions was at least as effective as a hospital clinic}

\author{
Fitzmaurice DA, Hobbs FD, Murray ET, et al. Oral anticoagulation management in primary care with the use of \\ computerized decision support and near-patient testing: a randomized, controlled trial. Arch Intern Med 2000 Aug \\ 14-28;160:2343-8.
}

QUESTION: For patients who require oral anticoagulation, is a nurse led clinic that
uses onsite blood testing and a computerised decision support system as effective as
routine hospital care for maintaining appropriate international normalised ratios (INRs)?

\section{Design}

Randomised \{allocation concealed $\}^{*}$, unblinded, controlled trial with 1 year of follow up.

\section{Setting}

9 of 21 potential general practices in Birmingham, UK.

\section{Patients}

224 adults (55\% men) who were receiving warfarin. Follow up was $82 \%$.

\section{Intervention}

122 patients were allocated to nurse led care in the general practice. The nurse met with the patient, measured the INR with onsite equipment, and used the computer program (Anticoagulation Management Support System [Softop Information Systems, Warwick, UK]) to direct dosing decisions. The program was based on the British Society of Haematology guidelines and had 2 main ranges of INR (2.0-3.0 and 3.0-4.5). If a dosing change was suggested, the nurse checked the change with medical staff at the clinic. 102 patients were allocated to hospital based care (2 of the 3 sites were staffed by a physician during the study and 1 site started with physician supervision before switching to a technician and another computer decision support program).

\section{Main outcome measures}

Proportion of patients who achieved appropriate INR control and mean percentage of time each patient spent within a therapeutic range of INR.

\section{Main results}

8 patients in the nurse led group returned to hospital clinic care. The groups did not differ for the proportion of patients who achieved appropriate INR control. Patients in the nurse led group had a higher percentage of time spent in the therapeutic INR range than patients in the hospital group $(69 \%$ v $57 \%, \mathrm{p}<0.001)$. The groups did not differ for all cause mortality ( 3 deaths in each group), serious adverse events (3 in each group), or overall adverse events. The mean intervention cost per patient was $£ 169$ in the nurse led group and $£ 69$ in the hospital group.

\section{Conclusion}

A nurse led clinic that included onsite blood tests and a computer decision support system for patients who were receiving warfarin was at least as effective as a hospital based clinic.

*Information provided by author.
Sources of funding: UK Medical Research Council Reaching Our Potential Award scheme UK National Health Service Research and Development Primary and Secondary Care Interface Programme.

For correspondence: Dr D A Fitzmaurice, Department of General Practice, Medical School, University of Birmingham, Birmingham B15 2TT, UK. Fax $+44(0) 121$ 4143759 .

A modified version of this abstract appears in Evidence-Based Medicine.

\section{COMMENTARY}

Managing patients on oral anticoagulation treatment is time consuming for the primary care provider. The frequent blood testing is disruptive to patients' daily lives. Despite considerable time and effort, studies confirm that patients are often outside their prescribed INR range. ${ }^{12}$ Because of the difficulties of the treatment, many patients who need anticoagulation are not treated.

The search for better methods for managing anticoagulation includes the use of computer decision support by physicians, nurses, or patients to reduce time and costs. The use of such software provides an algorithm, reduces disparities among providers in decision making, and increases adherence with care standards. ${ }^{3}$ The potential also exists for enhancing pattern recognition in individual patients, which could assist in the detection of interfering drugs or foods.

The study by Fitzmaurice $e t$ al is 1 of several that have attempted to verify reduced costs and increased effectiveness of oral anticoagulation treatment by using a combination of decision support software, moving testing closer to the patient (enhanced by the use of capillary devices for measuring prothrombin times or INR), and using nursing personnel or the patient as decision maker. ${ }^{1245}$ Most studies have confirmed enhanced adherence, improved outcomes, and reduced costs, especially if complications are considered in cost analyses. Fitzmaurice $e t$ al found that intervention costs were higher than control group costs because of the capital costs of setting up the practice based clinics and the increased frequency of testing in the intervention practices. Because the intervention included a complete package of care, it is not possible to determine which individual elements (eg, increased patient education by the nurses) contributed most to the positive results.

This study is of interest to nurses and nurse practitioners who may use such software. Oral anticoagulation management in primary care is an appropriate nursing function. The use of decision support software would provide the protocols that are legally required and would contribute to improved patient outcomes.

Stephanie Wright, RN, PhD, CFNP Associate Professor, Georgetown University Washington, DC, USA

1 Ansell JE, Patel N, Ostrovosky D, et al. Long term patient self-management of oral anticoagulation. Arch Intern Med 1995;155:2185-9.

2 Sawicki PT. A structured teaching and self-management program for patients receiving oral anticoagulation. JAMA 1999;281:145-50.

3 Lobach DF, Hammond WE. Computerized decision support based on clinical practice guidelines improves compliance with care standards. Am J Med 1997;102:89-98.

4 Lafata JE, Martin SA, Kaatz S, et al. The cost-effectiveness of different management strategies for patients on chronic warfarin therapy.J Gen Intern Med 2000;15:31-7.

5 Vadher B, Patterson DL, Leaning M. Evaluation of a decision support system for initiation and control of oral anticoagulation in a randomised trial. BMJ 1997;314:1252-6. 